\title{
Research on Intelligent Operation and Maintenance Platform Based on Automatic Calibration System of Electric Energy Meter
}

\author{
Rui Li, Peng-bo Shi, Ming-kai Li, Yuan Zhang, Ning Ding, He Wu \\ (State Grid Beijing Electric Power Company, Beijing, 100031, China)
}

\begin{abstract}
To the problem that the operation and maintenance of the electric energy meter automatic verification system is large and the equipment fault location not accurate, a smart operation and maintenance platform is developed. The function and structure of the system are analyzed, and the application effect of the intelligent operation and maintenance platform is demonstrated. The actual operation results show that: The application of intelligent operation and maintenance platform improves the operation and maintenance efficiency of the energy meter automatic verification system, which produces a good economic and social benefits.
\end{abstract}

\section{Introduction}

The energy meter is a measurement instrument for trade settlement between power supply companies, power generation companies and power customers. It is a nationally mandated electrical energy measurement instrument. According to the national measurement law, it must pass the laboratory verification before installation and use ${ }^{[1]}$. The conventional verification mode is manual work, which has the disadvantage of low work efficiency, high labor intensity, different verification levels and high business operating costs. It is difficult to meet lean management requirements.

The energy meter verification system consists of energy meter delivery system and verification device. Among them, the verification conveying system mainly includes feeding unit, conveying branch line unit, automatic connection and disconnection unit, image recognition unit, automatic sealing and marking unit, loading unit, abnormal loading unit, and empty box buffer unit; The verification device mainly includes withstand pressure verification device and multifunctional verification device. The various components are organically combined and tightly integrated through verification and transportation system to realize the entire process of intelligent, and automatic verification operations such as loading, transportation, verification, marking, boxing of the energy meter, as well as automation of non-stop operation in a pipelined manner verification system ${ }^{[3-4]}$.During the automatic verification process of the energy meter, it is necessary to inspect the operating status of the equipment, timely handle fault alarms, and timely replace damaged and aging components. At present, the automatic verification system of electric energy meters adopts the traditional manual operation and maintenance mode, and equipment failure handling is determined by experience. In the face of such a large-scale and complex production operation system, due to the work experience of each operation and maintenance personnel, there is a large difference in work capabilities, which often results in inaccurate fault location and long recovery time. The analysis of the cause of the failure by humans based on the failure phenomenon is prone to misjudgment, and the measures to deal with the failure are also based on personal experience. There is a large gap in the capacity of failure handling. If the core components of the verification device fail, which cannot be repaired in time, it will affect the verification efficiency. Manually record the failure analysis statistics of the verification system, with a large amount of data and inaccurate analysis statistics. Traditional operation and maintenance methods are relatively primitive, inefficient, and analysis data is easy to lose ${ }^{[5-6]}$. In order to improve the operation efficiency and operation and maintenance quality of the automatic verification system, it is possible to analyze the causes of equipment failure. Based on many years of operating experience of the automatic meter verification system, this paper integrates cutting-edge technologies such as workflow engines and the Internet of Things into the field of traditional metering operation and maintenance management system.

\section{System composition and functional characteristics of intelligent operation and maintenance platform}

\subsection{System composition of intelligent operation and maintenance platform}


The intelligent operation and maintenance platform system is mainly divided into equipment layer, communication layer, interface layer, application layer, and presentation layer. The theme frame of the system is shown in Figure 1 below. The equipment layer contains equipments such as AGV, multi-functional verification, and pressure-resistant devices. The communication layer is mainly for data interaction of mobile application APP, and communicates with the master station through "switch +LAN". The communication between the internal network and the external network considers security issues. The 95598 short-message platform with high security and maturity is used to send data short-messages to the external system's short-message cats in real time or time. The interface layer is responsible for the information interaction with the equipment system and the MDS system. It mainly obtains equipment file information, equipment fault information, production condition data, etc. The application layer mainly manages various closed-loop process orientations and analyzes various data. The presentation layer displays various business functions through web applications, mobile terminal applications, and Internet APP applications ${ }^{[7]}$.

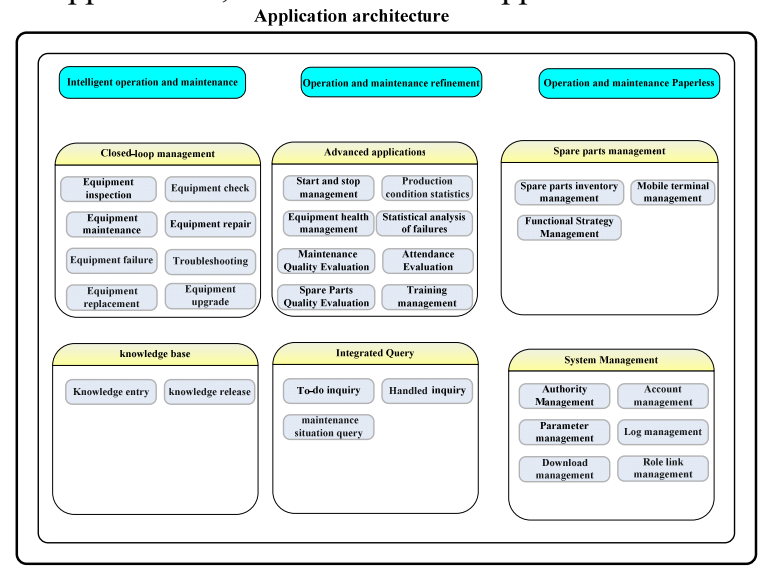

Fig.1 The frame of intelligent operation and maintenance platform

\subsection{Features of Intelligent Operation and Maintenance platform}

The business scope of the intelligent operation and maintenance platform includes data collection, comprehensive monitoring, operation and maintenance management, mobile operation management, knowledge base management, query statistics, and system management, as shown in Figure 2 below.

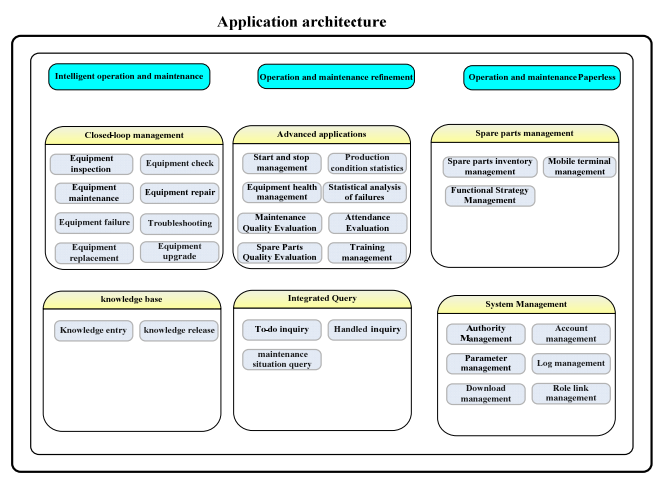

Fig.2 The application architecture of intelligent maintenance platform

The data acquisition module includes the collection of measurement production hardware information, the measurement production software information acquisition, MDS system operation related hardware information acquisition, MDS system operation related software information acquisition, operation and maintenance tool hardware information acquisition, operation and maintenance tool software information acquisition. The acquisition method mainly uses the interface method to collect and save the database for realtime display. The comprehensive monitoring module includes the system equipment operation status, operation and maintenance personnel, and production business conditions. The system can obtain the MDS system, various production systems and files related to the operation of related production facilities, operation information, personnel information, production plans, and production indicators to achieve Comprehensive monitoring of "four lines and one warehouse" fully automatic production operations. Mobile work management includes mobile work application management, mobile work equipment management, and corresponding application functions based on work roles. Equipment management mainly binds mobile operating equipment assets with the person responsible for receiving the goods based on the steps of storage, receipt, return, etc. Operation and maintenance management includes shift management, inspection management, troubleshooting management, cycle / special maintenance management, software upgrade management, spare parts management, and operation and maintenance quality assessment. Knowledge base management includes operation and maintenance experience database, knowledge entry, knowledge release, and knowledge application. Query statistics include system operation status query, system operation status evaluation, operation and maintenance service status query, and process work order query. The background data calculates the relevant results in real time/periodically to realize the statistical query of comprehensive data. System management includes security management, parameter management, and version management. Among them, the safety management is not less than the requirements of the secondary protection system. 


\section{Design and development of main function modules of intelligent operation and maintenance platform}

\subsection{Functional Requirements of Knowledge Base Management Module of Intelligent Operation and Maintenance Platform}

The intelligent operation and maintenance platform knowledge base mainly implements maintenance functions such as knowledge creation, modification, deletion, application planning, and management; knowledge systematic review and approval functions; and knowledge statistical analysis functions, including equipment abnormality analysis, fault processing situation analysis, maintenance situation analysis, Functions such as maintenance analysis, spare parts inventory analysis, etc .; which also have functions such as rights management, security level management, encryption, logging, and backup to protect the confidentiality, integrity, and effectiveness of knowledge; corresponding expansion interfaces should be provided for future systems Function expansion and integration.

\subsection{Establishment of knowledge management module of intelligent operation and maintenance platform}

The intelligent operation and maintenance platform knowledge base management module adopts layered management mode, and divides all operation functions into three layers: the knowledge base management layer, the knowledge base layer, and the knowledge base surface layer. Knowledge base management layer: It is used for various operations of knowledge base management. It directly calls the functions of the knowledge base layer. The knowledge base surface layer: the knowledge base surface layer function directly implements knowledge storage, query, modification, deletion and other operations in the knowledge base, it directly faces the database, the operation object is the underlying function. The design of the knowledge base should adopt an open technology platform for the overall design. It should support a variety of databases such as mysql, Sq1Server, oracle, and DB2, and have a smooth portability. It should adopt webservice, API and other interfaces to support the data layer, logic layer, and interface The integrated processing with user SSO has good scalability; security should be fully considered, and both the full sharing of information resources and the protection of information should be considered to ensure the security of the system.

\subsection{Application of Knowledge Base Management Module of Intelligent Operation and Maintenance Platform}

The application of the knowledge base management module mainly includes fault information processing, fault location, fault knowledge storage, fault knowledge base perfection, systematic state diagnosis, system maintenance planning, operation and maintenance assessment, etc. Fault information processing uses the fault knowledge base to retrieve equipment fault phenomena, determine equipment fault locations, and analyze and process them. The specific fault retrieval and fault processing process is shown in Figure 3. The fault location uses the search and query function of the fault knowledge base to input the fault phenomenon of the equipment, judge the fault cause of the equipment and locate the faulty components, and eliminate the equipment fault in time; the fault knowledge storage is based on the consistency, integrity and redundancy of the fault knowledge. The improvement of the fault knowledge base includes the correction of fault phenomena and fault location error information in the database, and timely addition of new fault phenomena, fault location, and fault processing rules to the fault knowledge base; systemic status Diagnosis Based on the analysis and statistics of equipment fault types, frequency, and outage time, systemically evaluate the equipment health status, and issue alarm alerts for equipment with higher frequency of failure; reasonably arrange inspection and maintenance plans based on the system's health status; For the equipment that issued the alarm, the cause of the failure should be analyzed and investigated in a timely manner, and eliminated in a timely manner. For equipment that is prone to failure and the frequency of the failure is high, the number of spare parts should be increased. Equipment from different manufacturers number, frequency of failures, system downtime and other data, as judge different manufacturers of equipment operation and maintenance of quality and quality of the merits of an important basis.

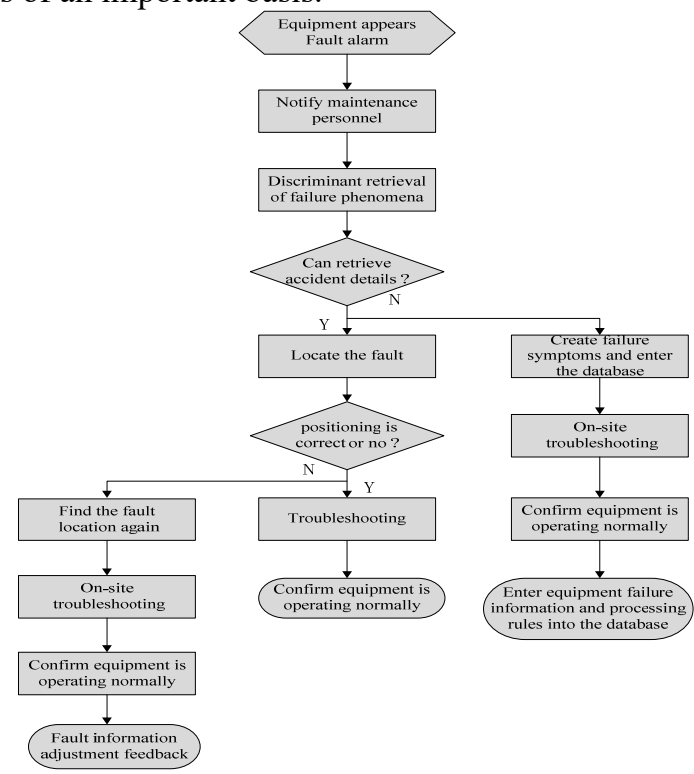

Figure 4 Intelligent maintenance platform main page display

\section{Functional verification of intelligent operation and maintenance platform}

After the development of the intelligent operation and maintenance platform, through continuous optimization 
and perfection, it has been successfully applied to the automatic verification system of energy meters, and its functions have reached the design requirements. The implementation of this project effectively improves the work efficiency of operation and maintenance personnel, improves the level of equipment operation and maintenance work supervision and control, and also provides an effective evaluation method for operation and maintenance personnel's operation and maintenance quality assessment.

\section{Benefit analysis of intelligent operation and maintenance platform}

\subsection{Economic Benefit Analysis of Intelligent Operation and Maintenance platform}

The application of the intelligent operation and maintenance platform can reduce the frequency of equipment failure, reduce equipment operation, operation and maintenance personnel costs, and the cost of spare parts, with significant economic benefits. Calculated according to a typical-scale enterprise (5 million annual energy meters verified), it can save funds in terms of equipment operation labor costs, equipment operation and maintenance labor costs, equipment operation and maintenance spare parts and cost, and the operation and maintenance costs have been reduced from 8.65 million yuan to 6 million yuan, saving 2.65 million yuan per year, and reducing operating costs by more than $30.6 \%$. The national annual verificating and testing of electrical energy measurement equipment is about 60 million, which can save operation and maintenance costs of 31.8 million. In summary, if the operation and maintenance platform can be promoted and applied across the country, the cost of energy meter manufacturing and application companies will be reduced to 58.8 million yuan, and the operating cost will be reduced by $37 \%$.On the other hand, after applying the intelligent operation and maintenance platform, the troubleshooting time can be shortened. Through online diagnosis and early warning technology, condition maintenance is carried out to reduce the frequency of failures, increase equipment availability, and ultimately increase production efficiency. Taking one provincial electric energy meter testing agency as an example, it runs 250 days per year, 8 hours a day, and the annual verification capacity is about 5 million units. After testing and verification, through intelligent operation and maintenance technology, the daily downtime of equipment failure can be reduced by 25 minutes, that is, the effective production time is increased by 25 minutes, and the verification quantity can be increased to 260,000 per year. Calculated based on the verification cost of 10 yuan per electric energy meter, the economic benefit reaches 2.6 million yuan.

\subsection{Social Benefit Analysis of Intelligent Operation and Maintenance Platform}

Through the implementation of the intelligent operation and maintenance platform, the "machine replacement" of daily operation and maintenance and the concentration of enterprise innovation capabilities are achieved, reducing operating costs and improving production efficiency, and ultimately driving the industry's overall manufacturing test intelligence level. At present, innovation in the direction of intelligent manufacturing has been carried out in various industries and fields, and gradually formed distinctive smart factories and digital workshops. However, the operation and maintenance management technology of smart factories and digital workshops is still relatively backward. Basically relying on professional on-site operation and maintenance, it is difficult to guarantee the operation and maintenance quality and operation and maintenance efficiency. The results of this project will lead the breakthrough development of digital workshop remote operation and maintenance technology in various industries.

\section{Conclusion}

An intelligent operation and maintenance platform was developed based on the problems in the operation and maintenance of the automatic meter verification system. The function characteristics, structure and structure of the intelligent operation and maintenance platform are analyzed, and its application effect is demonstrated. The actual operation results show that the application of the intelligent operation and maintenance platform improves the operation and maintenance efficiency of the automatic verification system of the energy meter, and produces better economic and social benefits.

\section{References}

1. Chu Da-hua. Electronic Energy Meter [M]. Beijing: China Electric Power Press, 2009.

2. Xiao Tao. Standardization of Intelligent Production System in Regional Metrology Center [D]. North China Electric Power University, 2013.

3. Zhang Yan, Huang Jin-juan, Xiao Tao, et al. Key technologies of metrology automated assembly line verification system [A]. Power System Technology, 2011, 35 (2): 657-661

4. Wang Yong, Yang Jin-feng. Design and Application of Energy Measurement Automation System for Large Power Supply Enterprises [J]. Electric Measurement \& Instrumentation, 2011 (11): 63-66

5. Huang Yang-jie, Liu Shui, Li Bin. Research on digital electric energy measurement and detection technology scheme $[\mathrm{J}]$. Electric Measurement \& Instrumentation, 2011 (04): 66-71

6. Liu Chao. Research on Automatic Inspection and Intelligent Diagnostic Technology of Four-line and One-bank System [J]. Engineering Technology, 2016.

7. Liao Chang-chu. PLC Basics and Applications [M]. Beijing: Machinery Industry Press, 2013 